\title{
Skin Wound Tension Reduction Device Combined with Ablative Fractional Carbon Dioxide Laser to Reduce Scar Formation After Excision of Pediatric Facial Skin Lesions: A Prospective Cohort Study
}

\author{
Haiting $\mathrm{Xu}^{1,2}$, Jing $\mathrm{Li}^{1}$, Zifu Zhou', Jianhai $\mathrm{Bi}^{1}$, Xiaoyang $\mathrm{Li}^{2}$, Ran Huo ${ }^{1,3}$ \\ 'Department of Plastic Surgery, Shandong Provincial Hospital, Cheeloo College of Medicine, Shandong University, Jinan, People's Republic of China; \\ ${ }^{2}$ Department of Hand and Plastic Surgery, The Second Affiliated Hospital and Yuying Children's Hospital of Wenzhou Medical University, Wenzhou, \\ People's Republic of China; ${ }^{3}$ Department of Plastic Surgery, Shandong Provincial Hospital Affiliated to Shandong First Medical University, Jinan, \\ People's Republic of China
}

Correspondence: Ran Huo, Department of Plastic Surgery, Shandong Provincial Hospital, No. 324, Jingwuweiqi Road, Huaiyin District, Jinan, 25002I, Shandong, People's Republic of China, Email huoran@medmail.com.cn

Purpose: To investigate the efficacy of skin wound tension reduction device (SWTRD) combined with ablative fractional carbon dioxide laser (CO2-AFL) for the prevention of scar formation following the excision of facial cutaneous lesions in children.

Methods: Patients undergoing surgical excision of facial cutaneous lesions in our hospital between May 2019 and April 2021 were enrolled. After the excision of facial cutaneous lesions and based on the personal intents and conditions, patients were assigned to undergo SWTRD combined with CO2-AFL. Outcome evaluations were as follows: defect size, incision width, scar width, the Vancouver Scar Scale (VSS) and University of North Carolina 4P Scar Scale (UNC4P).

Results: A total of 25 pediatric patients (mean age, 9.88 years) were enrolled in the study. Following the treatment of SWTRD+CO2AFL, scar widths were relatively narrow and the appearance of the incision scars was significantly improved. A significant reduction in the patient-reported UNC4P scores at 6 months $(3,1-4)$ was observed when compared with that at 2 months $(0,0-1)$ after surgery $(p<0.001)$. A similar reduction in the VSS scar scale was also evident (6 months: $1,0.75-2.5$ vs 2 months: $6.5-8.5 ; p<0.001)$.

Conclusion: Combined SWTRD and CO2-AFL treatment effectively modulates the scar formation after the incision is healed and resulting in preventing scar widening, leading to the improvement of scar appearance, reduction in wound pain and pruritus and its overall prognosis.

Keywords: facial cutaneous lesions, pediatric patients, skin wound tension reduction device, fractional carbon dioxide laser, scar

\section{Introduction}

Facial cutaneous lesions including congenital melanocytic nevus (CMN) and sebaceous nevus (SN) may adversely affect an individual's perception of attractiveness that leads to poor quality of life, ${ }^{1}$ in particular of those lesions on the cheeks. With advancing age, skin lesions progress and thus, surgical excision is the primary treatment for a CMN or SN on cheeks. $^{2}$

Following surgical excision, hypertrophic scars often ensue, which impacts the aesthetic appearance. ${ }^{3}$ It is generally understood that scar formation is a result of the excessive secretion of collagen and fibers during the repair process of the damaged area. ${ }^{4}$ The main risk factors for excessive scar formation include gender, genetics, age, skin type, injury type, cause of injury, wound size and depth, anatomical site and mechanical tension on the wound. ${ }^{5}$ Among these factors, mechanical wound tension has been shown to contribute significantly to healing and scar occurrence and development. ${ }^{6}$ Indeed, excessive wound tension from sutures may result in normal skin incision wounds, tissue necrosis, wound dehiscence, widening and hyperplasia of the scar at the edge of the incision. Therefore, reducing wound closing tension 
may contribute favorably to the prognosis of wound healing and appearance. In clinical practice, skin stretching closures have been widely used for the repair of skin and soft tissue injury. ${ }^{7}$ Studies have also shown that the application of a skin wound tension reduction device (SWTRD) on the postoperative incision inhibits the growth of scars. ${ }^{8}$

On the other hand, Ablative Fractional laser (AFL) is also an effective tool for treating scars, which improves the physical symptoms associated with the scar, ${ }^{9}$ Including those of atrophic acne scars, hyperplastic burn scars, surgical and trauma scars. ${ }^{10-12}$ Moreover, fractional laser treatment significantly improves the scar texture, color, pain, and itching when compared with conventional surgical methods or conservative treatments. ${ }^{13-17}$

Given the effectiveness of both the medical skin reduction closure technique and fractional laser in the prevention and treatment of scars, few studies have reported the combined application of these in clinical practice. Therefore, this study investigated the outcomes of applying ablative fractional carbon dioxide laser (CO2-AFL) in combination with SWTRD in preventing scars formation following the excision of facial skin lesions in children.

\section{Methods}

\section{Study Population}

This was a before-after cohort study. Pediatric patients aged 5 to 14 years old undergoing surgical excision for facial cutaneous lesions including congenital melanocytic nevus (CMN) or sebaceous nevus (SN) were enrolled. Patients were excluded from the enrollment if they were in a poor medical condition that precluded surgery under regional anesthesia. Other exclusion criteria included previous AFL treatment, wound infection, keloid scarring, comorbidities such as diabetes and contractive skin disorders (eg, scleroderma).

Patient demographics including age, body mass index (BMI), medical history, current medications, and adverse reactions (such as itching, blistering, red and swollen) were collected to determine any potential impacts these factors might have on scar outcomes.

\section{Study Approval}

This study was approved by the local ethics committee of the Second Affiliated Hospital and Yuying Children's Hospital of Wenzhou Medical University. Written informed consent was obtained from all participants and Parent or legal guardian of the study.

\section{Surgical Procedure}

Surgical excision of skin lesions and reconstruction were performed by two experienced surgeons. After preoperative evaluation and patient counseling, a fusiform block fashion was marked along the lesion with adequate excision margins. Next, local anesthesia was injected (1\% lidocaine with 1:100,000 epinephrine) and the incision was made along the designed curve with No. 11 scalpel. The range of undermining was kept at nearly the same distance as the diameter of the lesion. The skin incision was closed with a running cutaneous or interrupted suturing using 6-0 prolene sutures. Skin sutures were removed after approximately 7 days later.

Before applying the SWTRD, the skin surface was cleaned, incision above article gauze covering about $0.8 \mathrm{~cm}$ wide, cut skin wounds by graft loads to the right length, and then reduce graft loads symmetrically to reduce a set of both sides of the incision, and the tightening device of the lock (graft loads the initial reduction for five lock spacing, the gap width of $2.3 \mathrm{~cm}$ ), usually within $1 \sim 2$ lock, Tighten about $0.5 \sim 1.1 \mathrm{~cm}$ width, leaving a gap of $1.2 \sim 1.8 \mathrm{~cm}$ in the middle. If the incision radian was larger or angulated, the method of section paste can be applied (Figures 1 and 2). SWTRD (Lichen, Xinjin Shifeng Medical Instrument Co, Chengdu, China and Chuangbiwen, Haichuang Medical Instrument Co, Hangzhou, China) device is china Food and Drug Administration cleared for commercial use. The incision without the SWTRD was covered with ordinary gauze. SWTRD was used for at least 2 months after wound suturing or suture removal.

After suture removal, CO2-AFL surgery was performed, after optical $1 \%$ lidocaine cream was applied on scars for an hour for pain control, using the mode of Deep FX, $300 \mathrm{~Hz}, 5 \%$ density, no stacking. Based on the scar thickness, the parameters of CO2-AFL were altered to achieve the energy from $25 \mathrm{~mJ}$ to $50 \mathrm{~mJ} .{ }^{18}$ At the time of replace SWTRD, or 

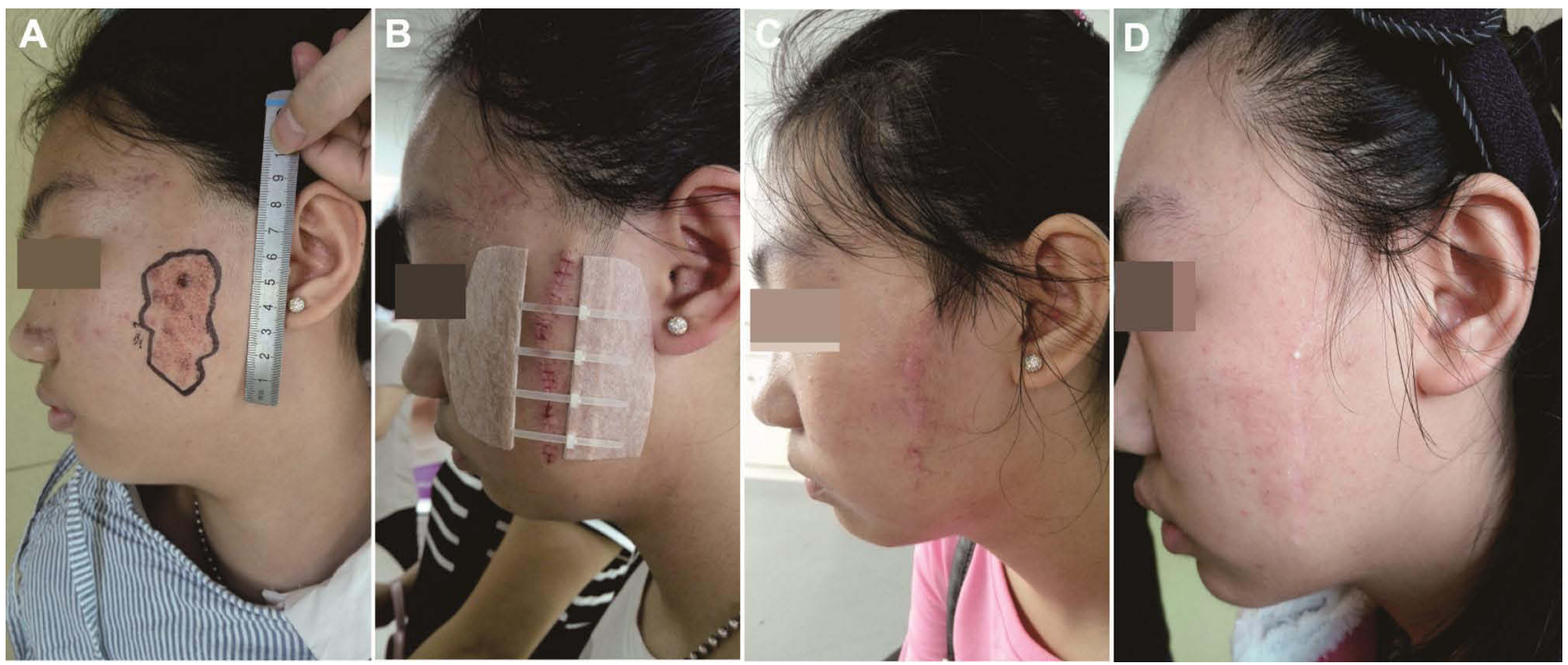

Figure I A 12-year-old female undergoing the excision of facial cutaneous lesions (sebaceous nevus, SN). (A) Before the excision of facial cutaneous lesions; (B) 7 days after the excision of facial cutaneous lesions; (C) 2 months after the excision of facial cutaneous lesions; (D) 6 months after the excision of facial cutaneous lesions.
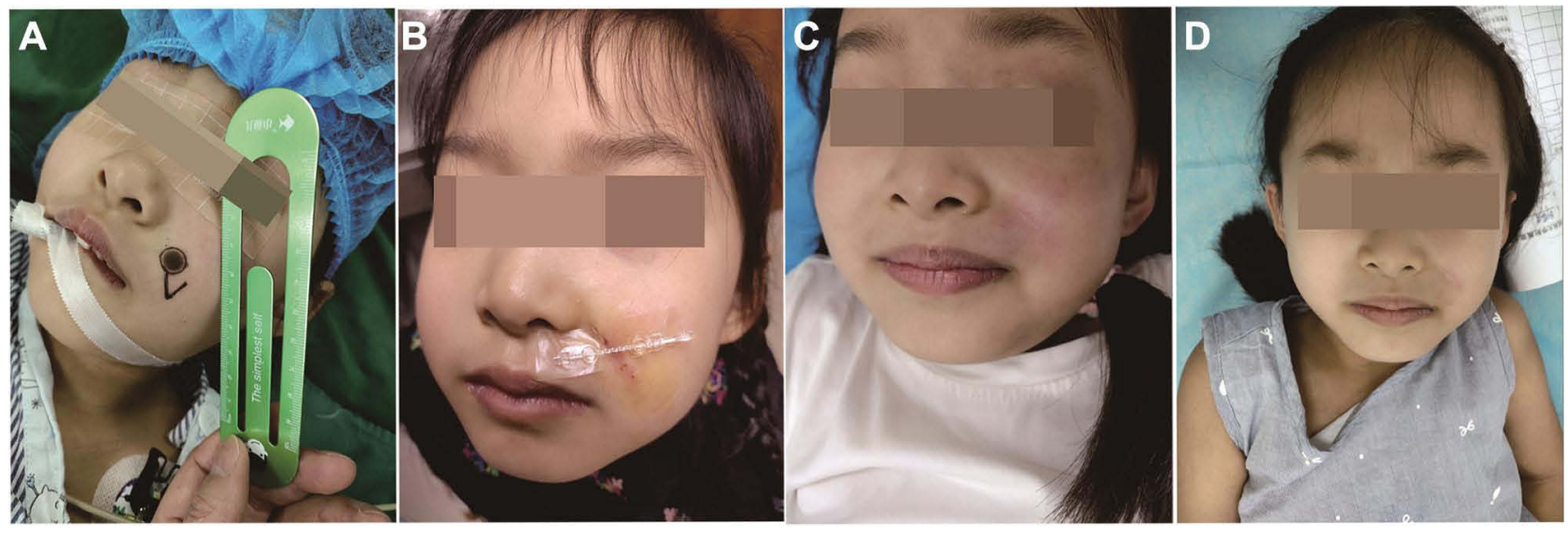

Figure 2 A 5-year-old female undergoing the excision of facial cutaneous lesions (congenital melanocytic nevus, CMN). (A) before the excision of facial cutaneous lesions; (B) 7 days after the excision of facial cutaneous lesions; (C) 2 months after the excision of facial cutaneous lesions; (D) 6 months after the excision of facial cutaneous lesions.

through the tensioner gap, CO2-AFL surgery is performed. Following every CO2-AFL surgery, wound gel (B. Braun Medical Inc, Bethlehem, PA) was applied for the following 7 days to promote epithelization. CO2-AFL was performed upon suture removal, at one month, 3 months, and 5 months later.

\section{Outcome Measurements}

The size of the skin lesion was measured before surgery.

Immediately after suture removal, about 14 days, and one month after the first and third CO2-AFL treatment, also at 2 and 6 months after surgery, the width of the scar was measured. For scar width, each group of incisions/scars was divided into four equal parts on average, and the three locations of $1 / 4,1 / 2$ and $3 / 4$ were selected to measure the width of scars using vernier calipers, and then the average value was taken for quantitative comparison.

One month after the first and third CO2-AFL treatment, also at 2 and 6 months after the surgery, the Vancouver scar scale (VSS) ${ }^{19}$ and the University of North Carolina 4P Scar Scale (UNC4P) ${ }^{20}$ of patients were assessed by 2 independent evaluators. The VSS assessed 4 variables: pigmentation, height/thickness, pliability, and vascularity. The scores of VSS ranged from 0 to 15 , with the highest score indicating the worst scar condition, while the score of 0 suggesting the best 
outcome. On the other hand, the UNC4P assessed 4 characteristics: pruritus, paresthesia, pain, and pliability. The scores of UNC4P ranged from 0 to 12 , with a higher score associated with more morbidity.

\section{Statistical Analysis}

Patients with complete follow-up data were analyzed using SPSS 21.0 (SPSS Inc., Chicago, IL, USA). Data were presented as mean $\pm \mathrm{SD}$, median, IQR, number and percentage. The scar widths were compared by paired, 2-tailed Student's $t$-test. VSS and UNC4P scores were compared by paired nonparametric test (such as Mann-Whitney $U$-test). A two-sided $\mathrm{p}$-value of $<0.05$ was considered statistically significant.

\section{Results}

\section{Patient Demographics and Baseline Characteristics}

A total of 25 pediatric patients with facial cutaneous lesions were included in our study (Table 1). Of these, 17 had CMN and 8 had SN. The means of age of participants were $9.88 \pm 2.77$ years old, BMI were $22.15 \pm 2.56$, scar length and scar width were $23.54 \pm 12.55 \mathrm{~mm}$ and $11.72 \pm 7.13 \mathrm{~mm}$, respectively. Complication rates associated with surgery were notably very low, One case each about red rash and blisters.

\section{Scar Widths}

At 7 days after surgery, the width of the wound was $0.28 \pm 0.10 \mathrm{~mm}$, the widths of scars after CO2-AFLsurgery, at 2 and 6 months were relatively narrow. At 6 months, the appearance of the scars was good, with the color and luster appeared similar to that of normal skin (Figures 1 and 2). The scar widths at 2 months and 6 months of treatment were 1.62 $\pm 0.68 \mathrm{~mm}$ and $1.68 \pm 0.70 \mathrm{~mm}$, respectively, which did not differ significantly (Table 2).

\section{Comparing the VSS and UNC4P Scores}

The global VSS scores at 6 months $(1,0.75-2.5)$ were significantly lower than that at 2 months $(6,5-8.5)$ after surgery $(\mathrm{p}<0.001$; Table 3$)$, with the pliability, vascularity, height and pigmentation scores of $(1,0-1 ; 0,0-1 ; 0,0-0.5 ; 0,0-1)$ and $(1,1-3 ; 2,1-2 ; 1,1-2 ; 2,1-2)$. These findings suggested that SWTRD+CO2-AFL could prevent scar formation (Table 3).

Table I Demographic and Baseline Characteristics of the Subset of Pediatric Patients

\begin{tabular}{|l|l|}
\hline & Patients (n=25) \\
\hline Male sex (N, percent) & $7(28 \%)$ \\
Female sex (N, percent) & $18(72 \%)$ \\
Age, years (mean \pm SD) & $9.88 \pm 2.77$ \\
BMI & $16.67 \pm 2.65$ \\
Lesion type (N, percent) & \\
CMV & $17(68 \%)$ \\
SGN & $8(32 \%)$ \\
Lesion length, mm (mean \pm SD) & $23.54 \pm 12.55$ \\
Lesion wide, mm (mean \pm SD) & $11.72 \pm 7.13$ \\
Length-to-width ratio of defect, (mean \pm SD, Range) & $2.30 \pm 1.32,1 . I-6.2$ \\
Wearing time of SWTRD, day (mean \pm SD) & $78.80 \pm 9.12$ \\
Adverse reaction (N, percent) & \\
Red rash & $1(4 \%)$ \\
Blisters & $1(1 \%)$ \\
\hline
\end{tabular}

Abbreviations: BMI, body mass index stands by weight/ height ${ }^{2}\left(\mathrm{~kg} / \mathrm{m}^{2}\right)$; $\mathrm{CMV}$, congenital melanocytic nevus; SGN, sebaceous glands nevus; SWTRD, skin wound tension reduction device; SD, standard deviation; $\mathrm{N}$, number. 
Table 2 Changes in Scar Width

\begin{tabular}{|l|l|l|l|l|}
\hline & 7 Days & 2 Months & 6 Months & P \\
\hline Width $(\mathrm{mm})$ & $0.28 \pm 0.10$ & $1.62 \pm 0.68$ & $1.68 \pm 0.70$ & 0.079 \\
\hline
\end{tabular}

Notes: Analysis width for 2 months compare to 6 months were performed with paired, 2-tailed Student $t$-test. Data are presented as mean \pm $\mathrm{SD}$

Abbreviation: SD, standard deviation.

Table 3 Changes in VSS $(n=25)$

\begin{tabular}{|l|l|l|l|}
\hline VSS & 2 Months & 6 Months & p \\
\hline Global score & $6,5-8.5$ & I, 0.75-2.5 & 0.000 \\
Pigmentation & I, I-3 & I, 0-1 & 0.000 \\
Vascularity & $2, I-2$ & $0,0-1$ & 0.000 \\
Height & I, I-2 & $0,0-0.5$ & 0.000 \\
Pliability & $2, I-2$ & $0,0-1$ & 0.000 \\
\hline
\end{tabular}

Notes: Analysis VSS for 2 months compare to 6 months were performed with paired MannWhitney- $U$ test. Data are presented as median.

Abbreviations: IQR, interquartile range; VSS, Vancouver Scar Scale.

On the other hand, the global UNC4P scores at 6 months $(0,0-1)$ were also significantly lower than that in 2 months $(3,1-4)$ after surgery $(\mathrm{p}<0.05$; Table 4$)$, with the pliability, pain, pruritus and paraesthesia scores of $(0,0-0.25 ; 0,0-0 ; 0$, $0-0 ; 0,0-0)$ and $(1,0.5-1 ; 1,0-1 ; 1,0-1 ; 0,0-1)$ respectively. Our findings suggested that the combined application of SWTRD+CO2-AFL improved scar outcomes effectively (Table 4).

\section{Improvements of UNC4P and VSS}

The scores of VSS and UNC4P at 6 months of treatment subtracting the scores at 2 months were used to evaluate the impact of the combined treatment. At 6 months, the total score on the VSS decreased by $4.86 \pm 1.87$. Pliability, vascularity, height and pigmentation scores decreased by $1.30 \pm 0.97,1.38 \pm 0.68,1.04 \pm 0.53$ and $1.14 \pm 0.57$, respectively (Figure 3). While the total score on the UNC4P decreased by $2 \pm 1.68$. Pliability, pain, pruritus and paraesthesia scores decreased by $0.24 \pm 0.42,0.66 \pm 0.61,0.58 \pm 0.71$ and $0.40 \pm 0.56$, respectively (Figure 4 ). These findings suggested that CO2-AFL combined with SWTRD comprehensively improved scar outcomes.

\section{Discussion}

The symmetry of facial appearance with minimal scar formations located at the boundary of the aesthetic subunit are expected favorable outcomes of facial reconstruction. ${ }^{21}$ A hypertrophic scar is a complication and is prevalent in approximately $64 \%$ of surgical incisions. ${ }^{22}$ Incision tension is crucially associated with scar formation. ${ }^{6}$ Studies have shown that local chronic inflammation caused by mechanical tension leads to immune response. Moreover, incision tension stimulates scar angiogenesis and collagen proliferation, reduces cell apoptosis, widens and aggravates scar, and

Table 4 Changes in NNC4P $(n=25)$

\begin{tabular}{|l|l|l|l|}
\hline UNC4P & 2 Months & $\mathbf{6}$ Months & $\mathbf{p}$ \\
\hline Global score & $3, \mathrm{I}-4$ & $0,0-\mathrm{I}$ & 0.000 \\
Pliability & $\mathrm{I}, 0.5-\mathrm{I}$ & $0.0-0.25$ & 0.000 \\
Pruritus & $\mathrm{I}, 0-\mathrm{I}$ & $0.0-0$ & 0.000 \\
Pain & $\mathrm{I}, 0-\mathrm{I}$ & $0.0-0$ & 0.002 \\
Paresthesias & $0,0-\mathrm{I}$ & $0.0-0$ & 0.003 \\
\hline
\end{tabular}

Notes: Analysis UNC4P for 2 months compare to 6 months were performed with paired MannWhitney- $U$ test. Data are presented as median.

Abbreviations: IQR, interquartile range; UNC4P, University of North Carolina 4P Scar Scale. 


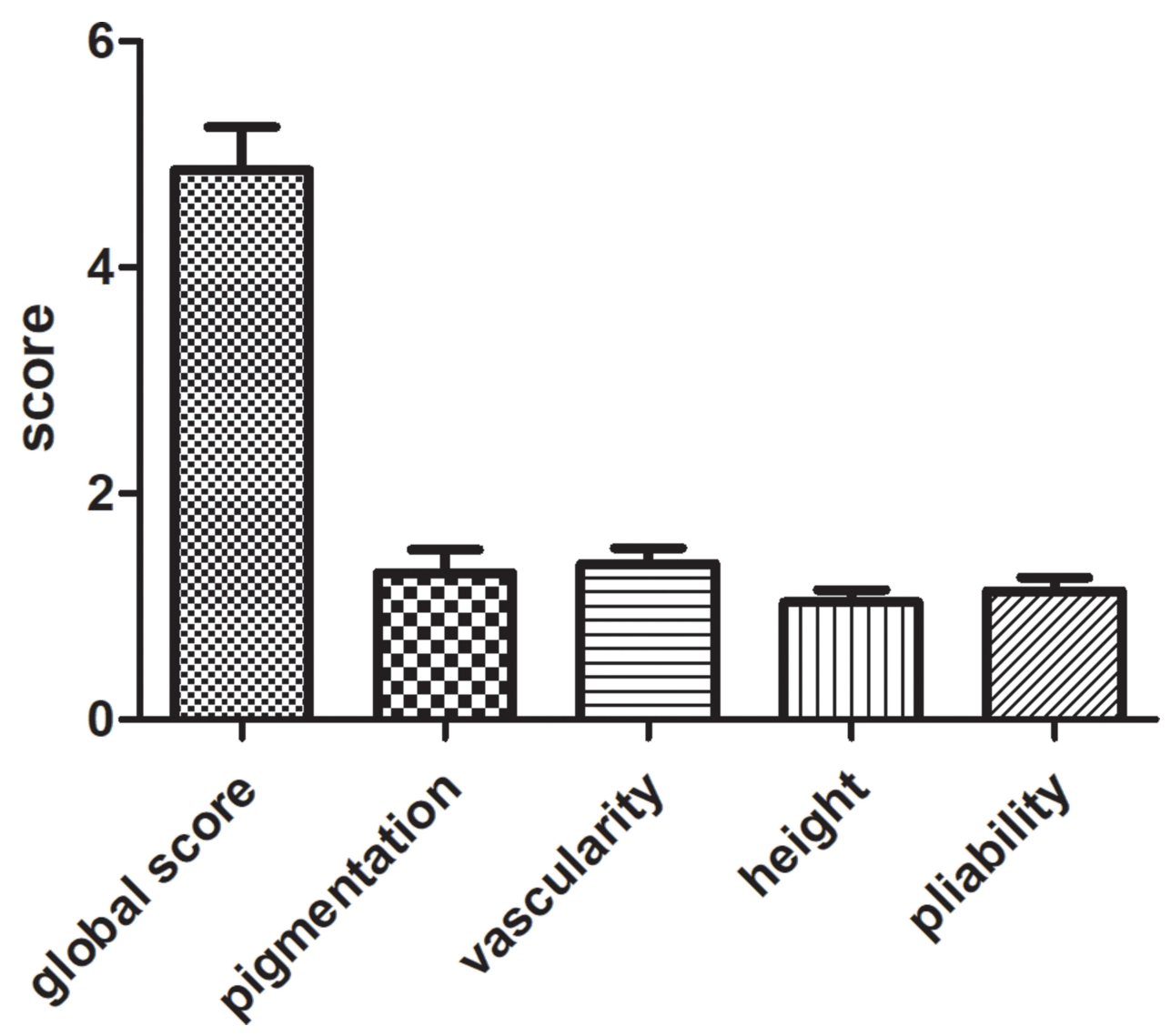

Figure 3 Improvement of pliability, vascularity, height and pigmentation as scored by the VSS at 6 months and 2 months following the AFL+SWTRD treatment. Mean with SD.

subsequently promotes scar proliferation. ${ }^{23,24}$ The presence of incision tension not only widens scars but also prolongs the immature period of scars. ${ }^{24-26}$ Basic laboratory research has demonstrated that reducing skin tension prevents the formation and development of hypertrophic scars. ${ }^{27}$ Thus, further clinical studies on strategies for reducing wound tension are warranted.

The SWTRD used in this study is a device that can be externally attached to the skin incision to transfer the tension of the wound to the normal skin away from the wound. Our analyses have revealed that the application of SWTRD resulted in a significantly lower VSS score and no change in scar width at 6 months than that of 2 months following surgery, indicating an effective reduction of the local tension of the early unhealed wound and later the healed wound, ultimately leading to inhibition of scar formation and suppressed scar proliferation (Figures 1 and 2) (Tables 2 and 3).

There are two types of externally applied tension reduction equipment, including the tension reduction tape and tension reduction device, both of which have been shown to improve the appearance of scars and reduce the incidence of scar hyperplasia. ${ }^{28,29}$ Now, there's a embrace advanced scar therapy device, consists of an applicator. It could reduce incisional scar formation. ${ }^{28}$ When applying the tension reducer, precautions should be taken to avoid the tension reduction strip being too tight that may result in excessive pressure on the surrounding skin. Although SWTRD is effective as a monotherapy to prevent excessive scar formation, for severe hyperplasia scar, combined physical therapy with other surgical treatments can be considered. Fractional lasers are a common form of therapy in the management of traumatic scars and surgical scars. Indeed, early laser intervention is beneficial in preventing traumatic scar formation by reducing the speed of scar development and enhancing the effectiveness of treatment response. In particular, the fractional laser treatment is effective in dissolving and eliminating small blood vessels, improving the flexibility and erythema of immature scars. ${ }^{30}$ In our study, after 6 months of CO2-AFL treatment, the measurements of VSS score revealed that the middle blood vessel score was lower than that at 2 months, indicating that the tensor combined with 


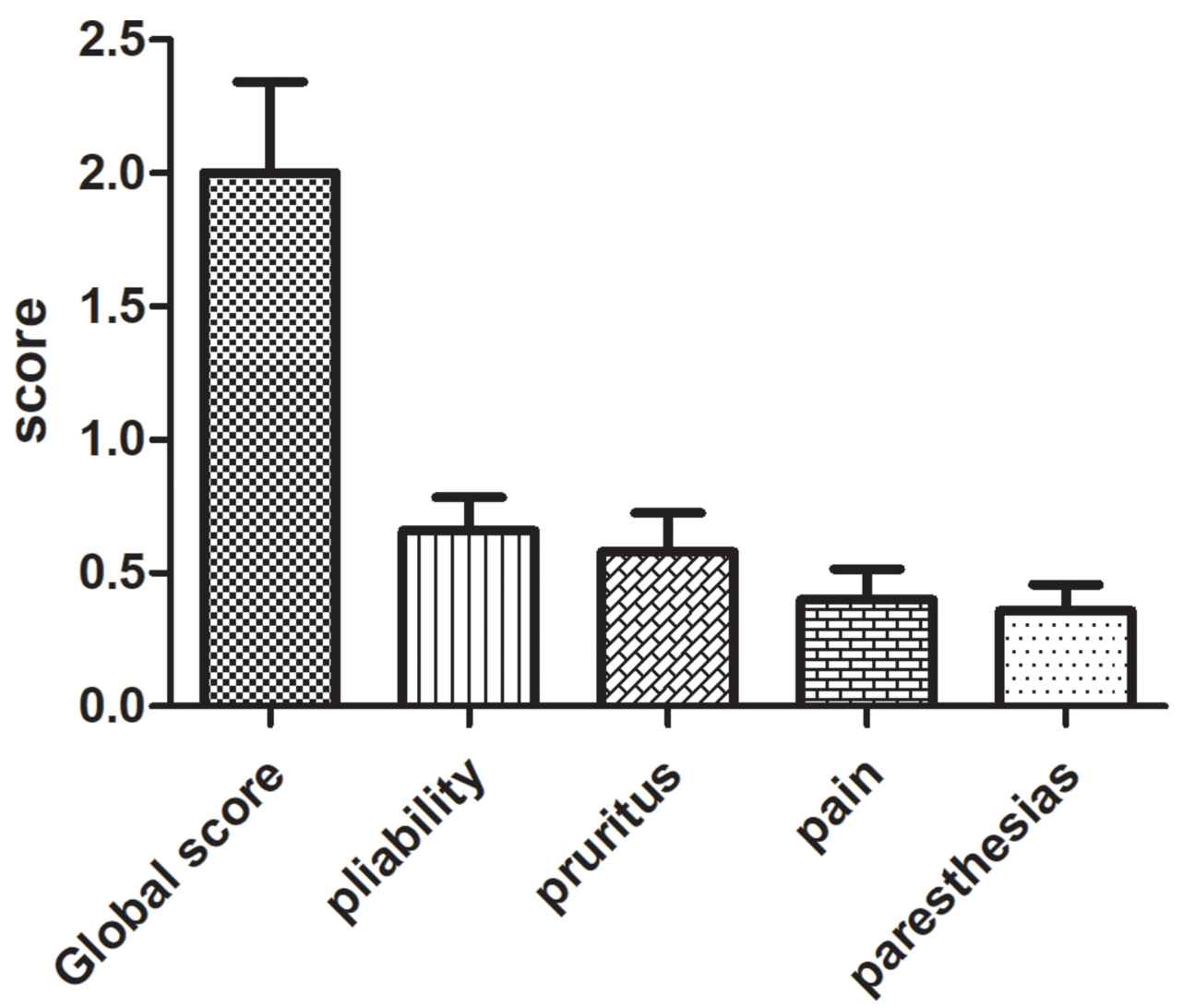

Figure 4 Improvements of pliability, pain, pruritus and paresthesia as scored by the UNC4P at 2 months and 6 months following the AFL+SWTRD treatment. Mean with SD.

laser treatment was effective in suppressing scar erythema (Table 3). Furthermore, our findings including narrow scar width at 2 and 6 months after surgery, the VSS score for all cheek scars was $(1,0.75-2.5)$ at 6 months and scoring from $(6,5-8.5)$, suggesting that scar hyperplasia was mild with rapid recovery. In addition, we observed that the scar pliability and height scores were $(0,0-1)$ and $(0,0-0.5)$, respectively. At 6 months, with some scars appeared mildly convex and firm, and some had only slight growth and soon resolving, indicating excellent outcomes of scar formation using our approach (Figures 1 and 2). Reductions in wound pain and pruritus were also observed (Table 4), likely attributed to the effects of CO2-AFL, which is consistent with the results of other studies. ${ }^{31}$ In generally, after three times CO2-AFL treatment, we find that VSS scores and NUC4P scores were low. This suggests that SWRTD combined with CO2-AFL could prevent scar formation.

It is accepted that the skin type of the majority of Chinese people is Fitzpatrick skin type 3-4, and the risk of scarring is relatively greater than in Caucasians. To date, continuous and multiple excision of the affected part is advocated as an effective treatment for $\mathrm{CMN}^{32}$ In our study, we excised the lesion only once, followed by the application of fractional laser and tension reducer to avoid wound dehiscence and prevent excessive scar formation. Therefore, our strategy advocates minimal surgery with one-off surgical excision that promotes recovery.

There were several limitations to our study. Firstly, the SWRTD had several drawbacks: some patients, especially the children, might experience discomfort when applying the device for an extended period of time; it appeared unsightly when applied on the face and needed to be replaced over time; uneven parts such as the nose and local skin with ulcers and infections would preclude the use of the device. Therefore, patients might be reluctant for long-term application of the device. Secondly, this study was limited to 6 months of postoperative management and follow-up. The tension reducer was removed within 3 months and thus, no comparative analysis was available with a shorter or a longer period of application of the device. Also, we could not determine and analyze different surgical sites in face. Maybe different surgical sites could due to different effects, which requires more detailed experiments. Thirdly, this study was a before-and-after single-cohort 
comparison with no control group. Finally, patients with a history of keloid were excluded from our study. Therefore, the effect of this treatment in the prevention of keloid remained unknown. Despite the limitations, our findings indicated that linear skin wounds were suitable for the application of a tension reduction retractor for at least 3 months after wound suturing or suture removal, in addition to 3 or CO2-AFL treatments based on the specific conditions of scars. This treatment could reduce postoperative scars and make postoperative scars look less obvious. Our analyses of favorable scar outcomes following the application of SWTRD combined with CO2-AFL warrant further validation from randomized control studies.

\section{Conclusion}

The SWTRD has a significant favorable impact on tension reduction of the surgical incision, which avoids excessive hyperplasia of the incision scar. CO2-AFL treatment of the incision effectively further reduces hyperplasia of the scar and blurs scar edge. Thereby combined SWTRD and CO2-AFL treatment effectively modulates the scar formation after the incision is healed and resulting in preventing scar widening, leading to the improvement of scar appearance, reduction in wound pain and pruritus and its overall prognosis.

\section{Abbreviations}

SWTRD, skin wound tension reduction device; CO2-AFL, combined with ablative fractional carbon dioxide laser; BMI, body mass index; VSS, Vancouver scar scale; UNC4P, University of North Carolina 4P Scar Scale; CMN, congenital melanocytic nevus; SN, sebaceous nevus.

\section{Ethical Approval}

The study protocol was approved by the local ethics committee of Second Affiliated Hospital of Wenzhou Medical University (LCKY2019-292) and with the 1964 Helsinki declaration.

\section{Informed Consent}

Informed consent has been obtained from the subjects (or their guardians) as specified in the ICMJE recommendations. Parent or legal guardian of all patients provided informed consent, and for Figures 1 and 2 to be published.

\section{Author Contributions}

All authors made a significant contribution to the work reported, whether that is in the conception, study design, execution, acquisition of data, analysis and interpretation, or in all these areas; took part in drafting, revising or critically reviewing the article; gave final approval of the version to be published; have agreed on the journal to which the article has been submitted; and agree to be accountable for all aspects of the work.

\section{Funding}

This research has been funded by Wenzhou Public Welfare Science and Technology Project (Y20170451), Wenzhou Basic Public Welfare Research Project (Y20210177). The funders had no role in the design, execution, or writing of the study. No other funding received.

\section{Disclosure}

The authors declare no conflicts of interest in this work.

\section{References}

1. Rankin M, Borah GL. Perceived functional impact of abnormal facial appearance. Plast Reconstr Surg. 2003;111(7):2140-2146. doi:10.1097/01. prs.0000060105.63335.0c

2. Young C, Han SS, Wook KB, et al. Effective treatment of congenital melanocytic nevus and nevus sebaceous using the pinhole method with the erbium-doped yttrium aluminium garnet laser. Ann Dermatol. 2014;26(5):651-653. doi:10.5021/ad.2014.26.5.651

3. Lv KY, Xia ZF. Chinese consensus panel on the prevention and treatment of scars. Chinese expert consensus on clinical prevention and treatment of scar. Burns Trauma. 2018;6:27. doi:10.1186/s41038-018-0129-9 
4. Gold MH, Mcguire M, Mustoe TA, et al. Updated international clinical recommendations on scar management: part 2-algorithms for scar prevention and treatment. Dermatol Surg. 2014;40(8):825. doi:10.1111/dsu.0000000000000050

5. Niessen FB, Spauwen P, Schalkwijk J, et al. On the nature of hypertrophic scars and keloids: a review. Plast Reconstr Surg. 1999;104 (5):1435-1458. doi:10.1097/00006534-199910000-00031

6. Chen H, Pan W, Zhang J, et al. The application of W-plasty combined Botox-A injection in treating sunk scar on the face. Medicine. 2018;97(30): e11427. doi:10.1097/MD.0000000000011427

7. Banner CN, Meadows WM. Examination of the effectiveness of various treatment techniques for reducing tension. Br J Clin Psychol. 2011;22 (3):183-193. doi:10.1111/j.2044-8260.1983.tb00599.x

8. Sun P, Ji Z, Li Z, et al. Prevention of scar hyperplasia in the skin by conotoxin: a prospective review. J Cosmet Dermatol. 2021;20(6):1885-1888. doi:10.1111/jocd.13761

9. Park KY, Oh IY, Seo SJ, et al. Appropriate timing for thyroidectomy scar treatment using a 1550-nm fractional erbium-glass laser. Dermatol Surg. 2013;39(12):1827-1834. doi:10.1111/dsu.12355

10. Oh BH, Hwang YJ, Lee YW, et al. Skin characteristics after fractional photothermolysis. Ann Dermatol. 2011;23(4):448-454. doi:10.5021/ ad.2011.23.4.448

11. Hurliman E, Zelickson B, Kenkel J. In-vivo histological analysis of a fractional CO2 laser system intended for treatment of soft tissue. $J$ Drugs Dermatol. 2017;16(11):1085-1090.

12. Anderson RR, Donelan MB, Hivnor C, et al. Laser treatment of traumatic scars with an emphasis on ablative fractional laser resurfacing consensus report. JAMA Dermatol. 2014;150(2):187-193. doi:10.1001/jamadermatol.2013.7761

13. Sorkin M, Cholok D, Levi B. Scar management of the burned hand. Hand Clin. 2017;33(2):305-315. doi:10.1016/j.hcl.2016.12.009

14. Elman S, Hynan LS, Gabriel V, et al. The 5-D itch scale: a new measure of pruritus. Br J Dermatol. 2010;162:587-593. doi:10.1111/j.13652133.2009.09586.x

15. Charles SH, Edkins RE, Cindy W, et al. Prospective, before-after cohort study to assess the efficacy of laser therapy on hypertrophic burn scars. Ann Plast Surg. 2013;70(5):521-526. doi:10.1097/SAP.0b013e31827eac5e

16. Hultman CS, Friedstat JS, Edkins RE, et al. Laser resurfacing and remodeling of hypertrophic burn scars: the results of a large, prospective, before-after cohort study, with long-term follow-up. Ann Surg. 2014;260(3):519-529. doi:10.1097/SLA.0000000000000893

17. Yun M, Jiang L, Yang H. The efficacy of fractional ablative carbon dioxide laser combined with other therapies in acne scars. Dermatol Ther. 2019;32(6):e13084. doi:10.1111/dth.13084

18. Karmisholt KE, Taudorf EH, Wulff CB, et al. Fractional CO2 laser treatment of caesarean section scars-A randomized controlled split-scar trial with long term follow-up assessment. Lasers Surg Med. 2017;49(2):189-197. doi:10.1002/1sm.22606

19. Baryza MJ, Baryza GA. The Vancouver scar scale: an administration tool and its interrater reliability. J Burn Care Rehabil. 1995;16(5):535-538. doi:10.1097/00004630-199509000-00013

20. Zhang N, Yu X, Zhao J, et al. Fractional CO2 laser therapy for cesarean scar under the guidance of multiple evaluation methods: a retrospective study. Cosmet Dermatol. 2020;20(7):2119-2124. doi:10.1111/jocd.13840

21. Yang J, Huang J, Chuang S. Long term follow up of burned face scar reconstruction using tissue expander. Burns. 2007;33(1):S130-S130. doi:10.1016/j.burns.2006.10.302

22. Gurtner GC, Werner S, Barrandon Y, et al. Wound repair and regeneration. Nature. 2008;453(7193):314-321. doi:10.1038/nature07039

23. Butzelaar L, Ulrich M, Mink V, et al. Currently known risk factors for hypertrophic skin scarring: a review. J Plast Reconstr Aesthet Surg. 2016;69 (2):163-169. doi:10.1016/j.bjps.2015.11.015

24. Gurtner GC, Dauskardt RH, Wong VW, et al. Improving cutaneous scar formation by controlling the mechanical environment: large animal and Phase I studies. Ann Surg. 2011;254(2):217-225. doi:10.1097/SLA.0b013e318220b159

25. Wong VW, Rustad KC, Akaishi S, et al. Focal adhesion kinase links mechanical force to skin fibrosis via inflammatory signaling. Nat Med. 2012;18 (1):148-152. doi:10.1038/nm.2574

26. Yagmur C, Akaishi S, Ogawa R, Guneren E. Mechanical receptor-related mechanisms in scar management: a review and hypothesis. Plast Reconstr Surg. 2010;126(2):426-434. doi:10.1097/PRS.0b013e3181df715d

27. Daegu S, Aram H. Overview of surgical scar prevention and management. J Korean Med Sci. 2014;29(6):751-757. doi:10.3346/ jkms.2014.29.6.751

28. Longaker MT, Rohrich RJ, Greenberg L, et al. A randomized controlled trial of the embrace advanced scar therapy device to reduce incisional scar formation. Plast Reconstr Surg. 2014;134(3):536-546. doi:10.1097/PRS.0000000000000417

29. Atkinson J-A, McKenna K, Barnett AG, et al. A randomized, controlled trial to determine the efficacy of paper tape in preventing hypertrophic scar formation in surgical incisions that traverse langer's skin tension lines. Plast Reconstr Surg. 2005;116(6):1648-1656. doi:10.1097/01. prs.0000187147.73963.a5

30. Alster TS, Nanni CA. Pulsed dye laser treatment of hypertrophic burn scars. Plast Reconstr Surg. 1998;102(6):2190-2195. doi:10.1097/00006534199811000-00060

31. Lv K, Liu H, Xu H, et al. Ablative fractional CO2 laser surgery improving sleep quality, pain and pruritus in adult hypertrophic scar patients: a prospective cohort study. Burns Trauma. 2021;9:tkab023. doi:10.1093/burnst/tkab023

32. Hassanein AH, Rogers GF, Greene AK. Management of challenging congenital melanocytic nevi: out comes study of serial excision. J Pediatr Surg. 2015;50(4):613-616. doi:10.1016/j.jpedsurg.2014.08.020 


\section{Publish your work in this journal}

Clinical, Cosmetic and Investigational Dermatology is an international, peer-reviewed, open access, online journal that focuses on the latest clinical and experimental research in all aspects of skin disease and cosmetic interventions. This journal is indexed on CAS. The manuscript management system is completely online and includes a very quick and fair peer-review system, which is all easy to use. Visit http://www. dovepress.com/testimonials.php to read real quotes from published authors.

Submit your manuscript here: https://www.dovepress.com/clinical-cosmetic-and-investigational-dermatology-journal 